\title{
Sur certains traits généraux de relations réciproques entre divers facteurs géographiques
}

\author{
Gaspard G. Mistardis
}

\section{Introduction}

$1^{\circ}$ Dans le cas d'une aire Méditerranéenne, dans laquelle un grand bras de mer parsemé de très nombreuses îles et entouré de terres très accidentées, comme l'aire égéenne, les relations réciproques entre les divers facteurs géographiques se présentent sous un jour extrêmement compliqué. Mais, elles nous donnent une image très intéressante, d'autant plus que cette aire présente de très grandes différentiations des conditions du milieu dans l'espace et dans le temps, et que, depuis plusieurs millénaires, le niveau de développement fut souvent extrêmement élevé.

Dans ce très court exposé, ne sont tracés que quelques aspects généraux de cette image.

\section{La géographie comme lien entre le passé et le proche} avenir

$2^{\circ}$ Etant donné que la géographie, en dehors de la représentation (cartes, etc.) et la description des caractéristiques de la surface du globe, cherche à expliquer leur origine et à tracer leur évolution probable, il s'ensuit qu'elle sert de lien entre le passé et le proche avenir quant à l'environnement et aux aspects de la vie.

Elle traite par conséquent des influences des conditions naturelles à la distribution spatiale de l'homme et de ses activités, de même que de l'intervention de l'homme dans le milieu physique, auquel il cause de multiples et diverses altérations.

Chaque fois que ces altérations sont très importantes, ce qui fut le cas souvent dans l'aire égéenne, le nouvel environnement physico-anthropogène exerce sur les hommes des influences sensiblement différentes des précédentes. Il s'ensuit donc que les influences réciproques entre le milieu et l'homme subissent dans le temps des changements appréciables.

$3^{\circ} \mathrm{La}$ géographie, dans le domaine de laquelle est comprise l'étude de ces influences réciproques n'étudie donc pas seulement l'état présent de la surface terrestre, mais elle s'avance aussi dans son passé et vers son avenir. L'aire égéenne est parmi celles qui sont les plus propres à mieux faire comprendre l'importance de ce lien avec le passé et l'avenir, surtout dans le domaine de la géographie humaine.

\section{Le déterminisme en géographie}

$4^{\circ}$ Dans le passé l'action de l'homme sur le milieu fut d'abord et pendant très longtemps minime; elle commença ensuite à devenir de plus en plus sensible en s'accroissant avec un rythme lent durant un très long espace de temps; enfin, à la suite d'une évolution technologique très remarquable, le rythme s'accéléra fortement, surtout depuis la première moitié du XIXe siècle.

Donc, dans le passé prédomine en géographie humaine un déterminisme fort, mais qui peu à peu perdit de sa prédominance, surtout depuis le siècle passé.

$5^{\circ}$ Durant les temps historiques, l'action de l'homme sur le milieu présente dans plusieurs aires des variations très importantes. Dans l'aire égéenne, elle est en forte augmentation à l'époque mycénienne, pour diminuer ensuite dans une très large mesure. L'intervention fut beaucoup plus vigoureuse aux temps helléniques, mais considerablement affaiblie durant certaines périodes du Moyen Age et de l'époque moderne.

Il s'ensuit donc qu'en géographie humaine le déterminisme devait présenter aux temps historiques des fluctuations assez sensibles.

$6^{\circ}$ Le milieu physique est sujet aussi à des changements dus à des causes naturelles, les uns plus ou moins périodiques (fluctuations cycliques), les autres non périodiques. Des premiers, les plus importants sont les fluctuations climatiques qui, à leur tour, provoquent des variations hydrologiques, édaphologiques et dans la végétation. Tous ces changements, plus ou moins cycliques, ne sont pas sans répercussions, soit favorables, soit défavorables sur $l^{\prime}$ homme et son économie.

$7^{\circ}$ Il est vrai qu'à notre époque l'évolution technologique permet de contrebalancer, plus ou moins, certains effets et répercussions provoqués par des changements dans l'environnement dus, soit à l'action de l'homme, soit à des causes naturelles. Mais, la plupart du temps, d'autres effets et répercussions défavorables suivent, soit peu après, soit plus tard.

$8^{\circ} \mathrm{Il}$ résulte de ce qui vient d'être exposé ici qu'en géographie, dans le domaine de laquelle l'étude des fluctuations, etc. déjà mentionnée $(\S 2,4-7)$ est comprise, le déterminisme doit tenir encore une place assez importante. 
La géographie comme science des inégalités et le principe de l'unité terrestre

$9^{\circ}$ A cause des différentiations spatiales très accusées dans l'environnement, sur la terre, la géographie est évidemment une science des inégalités, mais elle l'est aussi à cause du spectacle des diversités sociales dans l'espace.

$\mathrm{Ce}$ trait fondamental de notre discipline acquiert une ampleur plus grande à cause des variations dans le temps, tant des conditions du milieu dues, d'une part, à l'action de l'homme et, d'autre part, à des causes naturelles, que du spectacle des diversités sociales.

L'aire égéenne est une des plus propres dans le monde à faire mieux concevoir que la géographie est une science des inégalités dans l'espace et dans le temps.

$10^{\circ}$ En tout cas, ce trait des inégalités n'efface pas un autre trait fondamental de la géographie, soit le principe de l'unité terrestre tel qu'il a été conçu par Ratzel et Vidal de la Blache.

\section{Complications à cause des juxtapositions}

$11^{\circ}$ Les variations dans le temps des influences réciproques entre le milieu physico-anthropogène et les hommes deviennent plus compliquées, à la suite des juxtapositions des altérations dans l'environnement par l'action de l'homme avec les fluctuations des conditions de milieu dues à des causes naturelles. Il se peut ainsi que les effets et répercussions de l'action de l'homme sur le milieu dans une aire pourraient devenir moins défavorables si les changements dans le milieu dus à des causes physiques favorisaient l'œuvre restauratrice de la nature, ou s'aggraver au contraire si ces changements apportaient eux aussi d'autres effets défavorables.

$12^{\circ}$ Les différentiations spatiales du milieu physique sur la surface du globe ajoutent d'autres complications à celles dues aux juxtapositions mentionnées plus haut.

Il se peut ainsi que dans un pays nous aurions de ce fait des effets et répercussions favorables dans certaines régions, moịns favorables dans d'autres.

$13^{\circ}$ L'aire égéene est parmi celles qui se prêtent le mieux pour l'étude des problèmes qui surgissent des complications issues des juxtapositions.

\section{Complications issues des superpositions}

$14^{\circ}$ Souvent, durant des périodes parfois très longues, l'action de l'homme sur le milieu se ralentit extrêmement, à cause surtout d'une forte diminution de la population. Ce fut le cas plusieurs fois dans l'aire égéenne durant les quatre derniers millénaires.

Pendant ces périodes, la nature avait le temps de restaurer, au moins en partie, la plupart des altérations apportées à l'environnement par l'homme et rétablir ainsi plus ou moins les conditions du milieu à leur niveau antérieur. Mais, à ce qui reste des anciennes altérations, s'ajoute chaque fois ce qui reste des nouvelles. Il est évident qu'à cause de ces superpositions, la transformation du paysage devient de plus en plus profonde, mais pas en même degré partout à cause des différentiations spatiales de certains caractères (lithologiques, etc.) du milieu.

Il s'ensuit donc qu'à cause de ces superpositions, d'autres complications s'ajoutent au problème des influences réciproques entre le milieu et l'homme dans le temps. L'aire égéenne, dans laquelle les superpositions mentionnées plus haut sont très accusées, offre un champ propre pour l'étude de ces complications.

\section{Equilibre entre le milieu et l'homme}

$15^{\circ}$ Les besoins, de plus en plus grands provenant d'un développement économique en évolution rapide, conduisent à une action de plus en plus vigoureuse de l'homme sur le milieu. Il est naturel que les effets et répercussions défavorables qui ne tarderont pas à apparaître, feront naître des inquiétudes très sérieuses.

Il s'ensuit donc, que la nécessité d'un certain équilibre entre la nature et l'homme devient de plus en plus impérieuse. Mais, pour un tel équilibre, il faut d'une part des efforts plus vigoureux pour la conservation de la nature et de ses ressources, et d'autre part mettre un frein aux ravages des ressources naturelles et aux actions destructrices de l'homme.

$16^{\circ}$ Etant donné cependant qu'on ne peut pas ignorer les besoins du développement économique, il s'ensuit qu'il est surtout question de coordinations. Pour que ces coordinations puissent donner des résultats satisfaisants, il faut évidemment tenir compte du complexe extrêmement compliqué des influences réciproques entre la nature et l'homme, comme il a été exposé ici.

\section{Fondements géographiques des développements}

$17^{\circ}$ De ce qui vient d'être exposé dans cette étude très sommaire, il résulte que le développement d'une aire dépend non seulement de ses richesses naturelles et du niveau d'action de l'homme, mais aussi de la somme des effets et répercussions des influences réciproques entre le milieu et l'homme dans le temps et dans l'espace. 
Etant donné, que l'étude de ces influences réciproques est comprise dans le domaine de la géographie, il s'ensuit que les fondements d'un développement économique satisfaisant doivent être surtout géographiques ${ }^{1}$.

\section{Note}

1 Certains des problèmes, dont il est question dans cette très brève étude, sont traités plus en détail dans des travaux de l'auteur, mentionnés plus bas, qui se rapportent à l'aire égéenne:

a) Effets de l'érosion sur le déclin de la civilisation Mycénienne (VIIe Réunion technique de l'Union internationale pour la conservation de la nature et de ses ressources, C. R., vol. I, Bruxelles 1959, pp. 85-89).

b) Etudes géoéconomiques dans l'aire égéenne. I, L'île d'Andros (Bull. Soc. hellénique de Géographie, III, pp. 79-94, 167-213, 1959); en grec.

c) Crète. Problèmes agroéconomiques dans: Economie et Société, Athènes 1961, pp. 135-162); en grec.

d) Certains problèmes concernant l'aire Hellénique (Archives des Sciences économiques et sociales, V, 44, Athènes 1964, pp. 1-28); en grec. e) Cambios ecologicos y problemas de desarrollo (U.I. C. Conferencia regional latinoamericana, Mexico 1966, V, II, p. 26-37).

f) L'aire Sud-Egéenne. Changements dans le milieu géographique et cycles de développement économique très importants (Mélanges de Géographie, V, II, Gembloux 1968, pp. 253-276).

Wesenszüge der Wechselwirkungen zwischen verschiedenen geographischen Faktoren

Ausgehend vom Beispiel der ägäischen Region, ihrer im Laufe der Geschichte vielseitig sich verflechtenden natürlichen und kulturellen Faktoren, zeichnet der Aufsatz den wechselnden Einfluß der verschiedenen landschaftsbildenden Elemente auf die Entwicklung einer Gegend. Nicht bloß die Naturschätze und das kulturell-zivilisatorische Niveau des Menschen spielen dabei eine Rolle; vielmehr macht sich das gesamte raumzeitliche Gefüge der physio- und kulturgeographischen Auswirkungen darin geltend. Der Autor zieht daraus den Schluß, daß die Grundlagen für eine zufriedenstellende wirtschaftliche Entfaltung einer Region durch die geographische Wissenschaft bereitgestellt werden müssen. 